\title{
Experimental Validation of Simulations Using Full-field Measurement Techniques
}

\author{
Erwin Hack $^{\mathrm{a}}$ and Eann A. Patterson ${ }^{\mathrm{b}}$ \\ ${ }^{a}$ Laboratory Electronics/Metrology/Reliability, EMPA, Uberlandstrasse 129, CH-8600 Dübendorf, Switzerland, \\ erwin.hack@empa.ch \\ ${ }^{b}$ Composite Vehicle Research Center, Michigan State University, East Lansing, MI 48824, USA
}

\begin{abstract}
The calibration by reference materials of dynamic full-field measurement systems is discussed together with their use to validate numerical simulations of structural mechanics. The discussion addresses three challenges that are faced in these processes, i.e. how to calibrate a measuring instrument that (i) provides full-field data, and (ii) is dynamic; (iii) how to compare data from simulation and experimentation.
\end{abstract}

Keywords: Calibration, Validation, Finite Elements, Strain, Displacement, Vibration, Optical testing, Camera. PACS: 06.20.fb; 42.62.Eh; 07.10.Pz; 42.87.-d ; 06.30.Bp ; 07.60.Ly

\section{INTRODUCTION}

While much effort has been undertaken to promote the idea of measurement uncertainty in the last decade, confusion is still around regarding the concepts of traceability, calibration or validation. The International Vocabulary of Metrology (VIM) [1] defines validation as "provision of objective evidence that a given item is adequate for an intended use". Hence, validation prevents the use of a measurement system which is inappropriate for the intended use. The adequateness of a strain or vibration measurement system for use in experimental mechanics involves ensuring its appropriateness for the level of deformation that is expected, for the necessary spatial resolution and for the measurement rate. When using measurement equipment the calibration of the instruments is a pre-requisite for validation. VIM defines calibration as the "operation that, under specified conditions, in a first step, establishes a relation between the quantity values with measurement uncertainties provided by measurement standards and corresponding indications with associated measurement uncertainties and, in a second step, uses this information to establish a relation for obtaining a measurement result from an indication." Often, the first step alone in this definition is perceived as being calibration. The calibration of the measuring instrument is the first link of the traceability chain which relates the measurement result to a reference through a documented unbroken chain of calibrations, each contributing to the measurement uncertainty (VIM). We will apply these concepts to reference materials for calibrating optical measurement instruments, focusing on two challenges: full-field and dynamic data. The motivation of this work is the common use of optical measurement systems for dynamic measurements such as vibrations and impacts which are safety relevant events in vehicles of all kinds. This challenge adds still another degree of freedom to the calibration process: the time. Since testing of dynamic events such as impacts is a cost intensive venture, the replacement of actual experimental testing by reliable, validated simulations is a goal of many developers. Although in engineering modelling the analysis of dynamic events has progressed well, an experimental validation of the numerical results is still needed [2].

To develop procedures for the experimental validation of numerical simulations and for the calibration of instruments that measure dynamic displacement or strain the European project ADVISE was started under the $7^{\text {th }}$ Framework Programme's Transport Scheme [3]. ADVISE addresses experimental validation of simulations of dynamic events using full-field optical methods with a focus on fibre reinforced polymers. The objectives of this project are to address complex dynamic analysis and to use full-field data from experiments to validate in a quantitative manner numerical simulations. This includes

CP1253, The $9^{\text {th }}$ International Conference on Vibration Measurements by Laser and

Noncontact Techniques and Short Course edited by E. P. Tomasini

(C) 2010 American Institute of Physics 978-0-7354-0802-9/10/\$30.00 
- Development of reference materials for calibration of full-field optical measurements of deformation in dynamic events;

- Optimisation of methodologies for both optical measurement and computational modelling and simulation of linear modal vibrations and non-linear impact responses;

- Contributions to standardisation activity for experimental validation of dynamic simulations.

\section{CALIBRATION AND REFERENCE MATERIALS}

When calibrating an instrument, most often a single reference quantity is captured by a single transducer and compared to the reading from the instrument. A linear variable differential transformer (LVDT) can be calibrated with a laser interferometer which serves as the traceable reference standard. In vibration analysis, reference accelerometers or heterodyne interferometers are common reference standards. The calibration of full-field techniques and the assessment of the corresponding measurement uncertainty is more involved, as the reference material must provide a field of reference values. To calibrate the imaging properties of a lens representative patterns (e.g. chequerboard pattern, USAF target, Siemens star) are popular. Similarly, for full-field techniques of strain, displacement or vibration measurement the need arises for an artefact that allows a full-field calibration of the quantity in question. The realization of such an artefact for static strain measurement was a main output of the European SPOTS project [4], and its use for calibrating an optical instrument is described elsewhere [5, 6]. The artefact is based on a monolithic frame including a beam under four point bending. The system has a simple analytic relationship between the traceable quantity (the deformation displacement of the frame) and the derived quantity to be calibrated (the strain field in the calibration area). To overcome the limitations of a static calibration in order to calibrate instruments for dynamic measurements of displacement or strain a design process was initiated with a poll of the experimental mechanics community conducted by the ADVISE project on the essential and desirable attributes of a reference material. The essential attributes include:

- reproducible boundary conditions;

- robustness and portability;

- availability of a range of displacement values inside the field of view;

- use for out-of-plane and in-plane displacements;

- data can be extracted throughout a cycle; and

- a means of verifying the performance in situ.

An embodiment of a reference material is under construction [7]. As in the static case, the deformation field will be verified by an in situ measurement, preferably at a single or a few points. These point measurements are used for establishing the traceability of the deformation data to the primary standard of length. In addition, a sinusoidal excitation will be used to calibrate the temporal aspects of the dynamic measurement system. A Laser Doppler Vibrometer could be helpful in characterizing the reference material or to verify its performance in situ.

\section{EXPERIMENTAL VALIDATION}

Figure 1 shows a schematic flow chart for a typical experimental validation procedure from the viewpoint of the different coordinate systems involved. The actual physical object (central strand) is under study and is embedded in the world coordinate system $(x, y, z)$. Neither its actual geometry and material properties nor its behaviour under load are known. We assume that there exists a numerical model and a simulation of its behaviour (left strand) as well as measurement results from the experiment (right strand), here symbolically denoted by a set of camera images. Both simulations and measurements will provide results, here represented by a strain component $\varepsilon$.

The first step consists in establishing the reference frames for the three strands. Harmonization of boundary conditions is a pre-requisite for a meaningful procedure. The step includes the definition of the quantity under test. For linear elastic experiments natural choices are displacement components or components of strain / stress. For destructive testing several approaches can be taken [8]. It is common use to characterize the geometrical extent or size of the damage, e.g. by visual, ultrasonic, radiographic or optical testing. While this can give valuable information, ADVISE is following two other approaches. One is concerned with the validation of the dynamic 


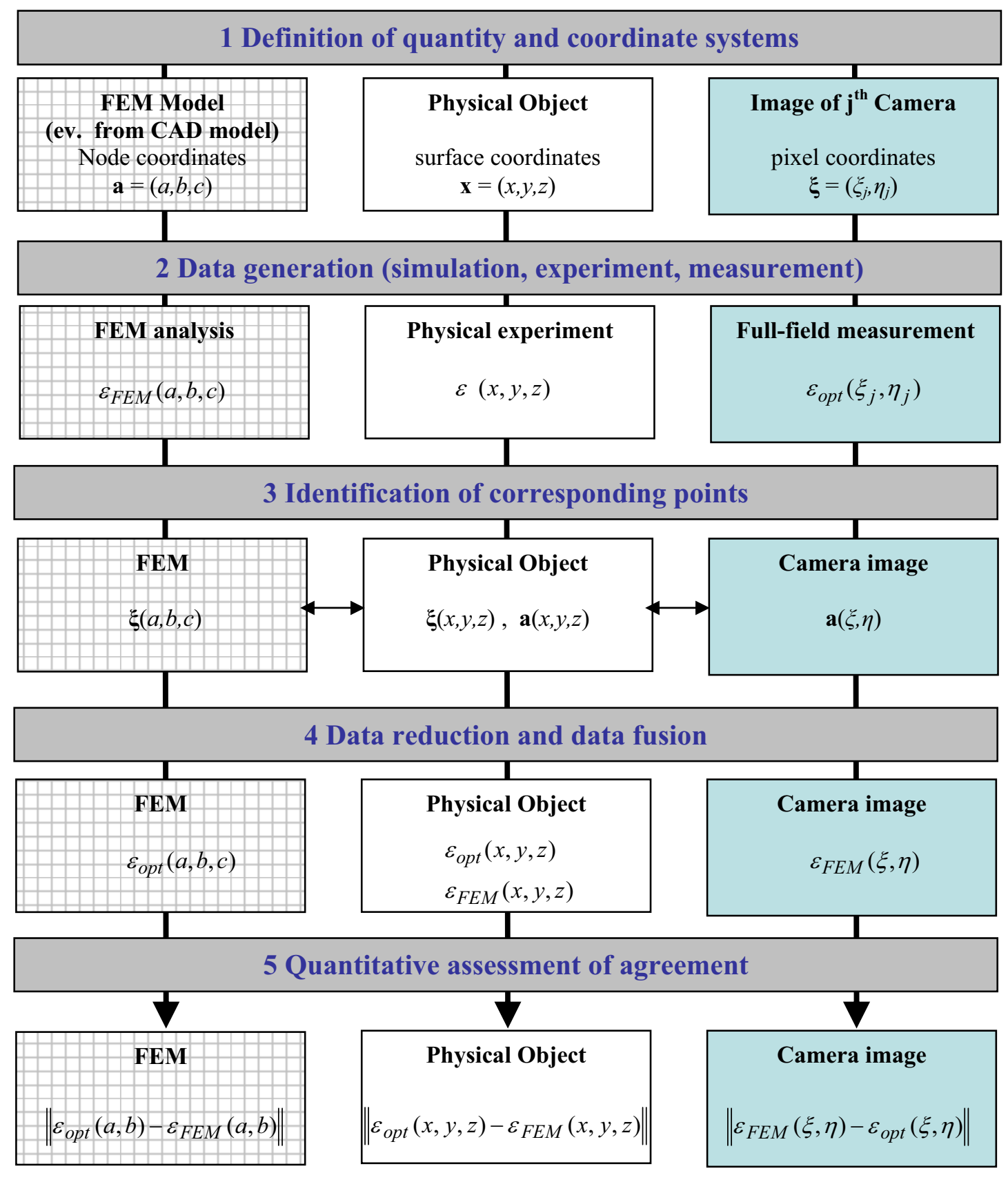

FIGURE 1. Flowchart of a typical experimental validation procedure. $\varepsilon$ is symbolic for the quantity of interest which could be a component of strain, but also of displacement or amplitude.

simulation. In this approach, the material non-linearities must be incorporated, and a dynamic experimental test must be performed. The example of impact testing on honeycomb panels is reported elsewhere [9]. The second ADVISE approach is centred on characterizing the effect damage has on the structure, and involves the comparison of the structural behaviour of a damaged sample with that of an undamaged sample .

During the second step in Figure 1 the experiment is performed, measurements are taken, and simulation results are generated. The physical experiment can again be a linear-elastic test, a damage-inducing event or a different appropriate test for characterizing the quantity of interest. Each result is expressed in the respective reference frame, as denoted by the expressions $\varepsilon_{F E M}(a, b, c)$ for the simulation results, $\varepsilon_{o p t}\left(\xi_{j}, \eta_{j}\right)$ for the optical measurement 
result of camera $\mathrm{j}$, and $\varepsilon(x, y, z)$ the actual experimental outcome that is to be characterized. As sketched above, the results can include a time dependence when measurements are taken during a dynamic event, and simulations are performed accordingly.

\section{DATA COMPARISON}

For validation of the numerical model the data from the simulation and experiment must be compared. Hence, the data must be converted into a common frame of reference. This is called "identification of corresponding points" in the third step. Symbolically, the coordinates of the camera are expressed in terms of the node coordinates of the numerical model $\xi(a, b, c)$ or vice versa, $\mathbf{a}(\xi, \eta)$. However, it is also possible to choose the object (or world) coordinates as the reference frame. Once the correspondence of the coordinate points is established, the results of the measurements and simulations are expressed in the common reference frame. Such procedures commonly involve data interpolation and data smoothing routines. Some optical measurement software allow the extraction of displacement and strain data from load state images for the node points of the finite element mesh that has been prepared for the object under investigation with the help of a FE pre-processor

For comparison, data are normally reduced in step four. Sometimes only a few selected values such as a maximum stress value or its location are of interest. Sometimes data are compared along a line. In both cases a quantitative comparison is mostly based on taking the difference of the data point by point and / or by calculating an average or maximum deviation. While this can give enough insight into selected aspects of the structural behaviour, it is far from being complete because most data points are ignored. Instead of extracting data from a line or grid of equidistant measurement points all available data ("full-field") should be considered in the evaluation. For a direct comparison with numerical calculations, the optical measurement data are therefore transformed into the nodes of the FEM meshes, whenever the optical data are more dense than the simulation data.

There exist several measures to obtain a quantitative figure of merit, such as the Modal Assurance Criterion (MAC) [10], simply given by the normalized scalar product of all $N$ simulated data points with the corresponding optical measurement data, and the rms criterion based on the root mean square deviation of the $N$ data points:

$$
\begin{gathered}
M A C=\frac{\left|\boldsymbol{\varepsilon}_{\text {opt }} \cdot \boldsymbol{\varepsilon}_{F E M}\right|^{2}}{\left|\boldsymbol{\varepsilon}_{\text {opt }} \cdot \boldsymbol{\varepsilon}_{\text {opt }}\right|\left|\boldsymbol{\varepsilon}_{F E M} \cdot \boldsymbol{\varepsilon}_{F E M}\right|} \in[0,1] \\
r m s=\frac{1}{N} \sqrt{\left|\boldsymbol{\varepsilon}_{\text {opt }}-\boldsymbol{\varepsilon}_{F E M}\right|^{2}}
\end{gathered}
$$

Both criteria can be expanded to include a weighting function. On the other hand such full-field point by point comparisons are not selective enough, and tedious, because all the point by point correspondences must be established before being able to apply the criteria.

It is therefore highly desirable to develop a procedure that takes into account the whole field of data, but at the same time reduces the number of necessary comparison points. This can be achieved by parameterising the measurement data and simulation results and comparing the parameter values. This approach is taken in ADVISE and several promising applications have been reported. Wang et al. [11] employed Zernike moments to describe modal shapes for the purpose of mode shape recognition and updating of a finite element model, while Patki et al. [12] use image decomposition as a tool for validating stress analysis models.

\section{CONCLUSIONS}

The comparison of experimental and numerical data is ubiquitous in the experimental mechanics community. Validation refers to the fitness for the intended use. Hence, it is the user who decides on the level of acceptable agreement of the data comparison. Nevertheless it is worth giving a quantitative assessment for the match of two data sets, as outlined above. We have discussed the need for developing procedures for calibration and validation of imaging full-field measurement systems for strain and displacement in view of the challenges posed by full-field and dynamic data. We have sketched the objectives of the European project ADVISE, among which are the development of a dynamic reference material for full-field calibration and the development of comparison strategies for validation of results from numerical model analysis. 


\section{ACKNOWLEDGMENTS}

Part of this work has received funding from the European Community's Seventh Framework Programme under Grant Agreement no. SCP7-GA-2008-218595. We thank our project partners for their valuable input and discussions: Richard Burguete (Airbus UK), Thorsten Siebert and Reinhard Schubach (Dantec Dynamics GmbH), Andrea Davighi and Maurice Whelan (EU Joint Research Centre), John Mottershead (University of Liverpool), George Lampeas (University of Patras), Alexander Ihle (High Performance Space Structure Systems GmbH), and Andrea Pipino (Centro Ricerche Fiat).

\section{REFERENCES}

1. ISO/IEC GUIDE 99:2007, International vocabulary of metrology - Basic and general concepts and associated terms (VIM).

2. ASME V\&V 10-2006, Guide for verification and validation in computational solid mechanics, American Society of Mechanical Engineers, New York, 2006.

3. ADVISE - Advanced Dynamic Validations using Integrated Simulation and Experimentation, FP7 project SCP7-GA-2008218595. see http://www.dynamicvalidation.org

4. SPOTS - Standardisation project for optical techniques of strain measurement, EU contract no. G6RD-CT-2002-00856, see http://www.opticalstrain.org

5. E. Hack, G. Sims, D. Mendels, "Traceability, reference materials and standardised tests in optical strain measurement", Proc. ICEM 12, 29 August - 2 September, 2004, Bari, Italy

6. E.A. Patterson, E. Hack, P. Brailly, R.L. Burguete, Q. Saleem, T. Siebert, R.A. Tomlinson, M.P. Whelan, "Calibration and evaluation of optical systems for full-field strain measurement", Opt. Las. Eng. 45, 550-564 (2007)

7. A. Davighi, E.A. Patterson, M. Whelan, "A reference material for calibration of dynamic displacement and strain measurements", Proc. ICEM 14, Poitiers (FR), 4 - 9 July 2010, paper 305

8. M. Feligiotti, E. Hack, and G. Lampeas, "Methodology for assessing impact damage using integrated simulation and experimentation", Proc. ICEM 14, Poitiers (FR), 4 - 9 July 2010, paper 332

9. G. Lampeas and T. Siebert, "Validation of non-linear dynamic simulations through full field optical methods", Proc. ICEM 14, Poitiers (FR), 4 - 9 July 2010, paper 321.

10. R.J. Allemang, "The modal assurance criterion - twenty years of use and abuse", Sound and Vibration Magazine 37, 14-23 (2003).

11. W. Wang, J. Mottershead, C. Mares, "Mode-shape recognition and finite element model updating using the Zernike moment descriptor", Mechanical Systems and Signal Processing 23, 2088-2112 (2009)

12. A. Patki, V. Wang, J. Mottershead, and E.A. Patterson, "Image decomposition as a tool for validating stress analysis models", Proc. ICEM 14, Poitiers (FR), 4 - 9 July 2010, paper 271 